\title{
Numerical Simulation of Roughness-Induced Transient Growth in a Laminar Boundary Layer
}

\author{
Paul Fischer ${ }^{*}$ \\ Argonne National Laboratory, Argonne, IL 60439 \\ and \\ Meelan Choudhari \\ NASA Langley Research Center, Hampton, VA 23681
}

\begin{abstract}
Numerical simulations are used to examine the roughness-induced transient growth in a laminar boundary-layer flow. Based on the spectral element method, these simulations model the stationary disturbance field associated with a nonsmooth roughness geometry, such as the spanwise periodic array of circular disks used by White and co-workers during a series of wind tunnel experiments at Case Western Reserve University. Besides capturing the major trends from the recent measurements by White and Ergin, the simulations provide additional information concerning the relative accuracy of the experimental findings derived from two separate wall-finding procedures. The paper also explores the dependence of transient growth on geometric characteristics of the roughness distribution, including the height and planform shape of the roughness element and the ratio of roughness size to spacing between an adjacent pair of elements. Results are used for a preliminary assessment of the differences between recently reported theoretical results of Tumin and Reshotko and the measurements by White and Ergin.
\end{abstract}

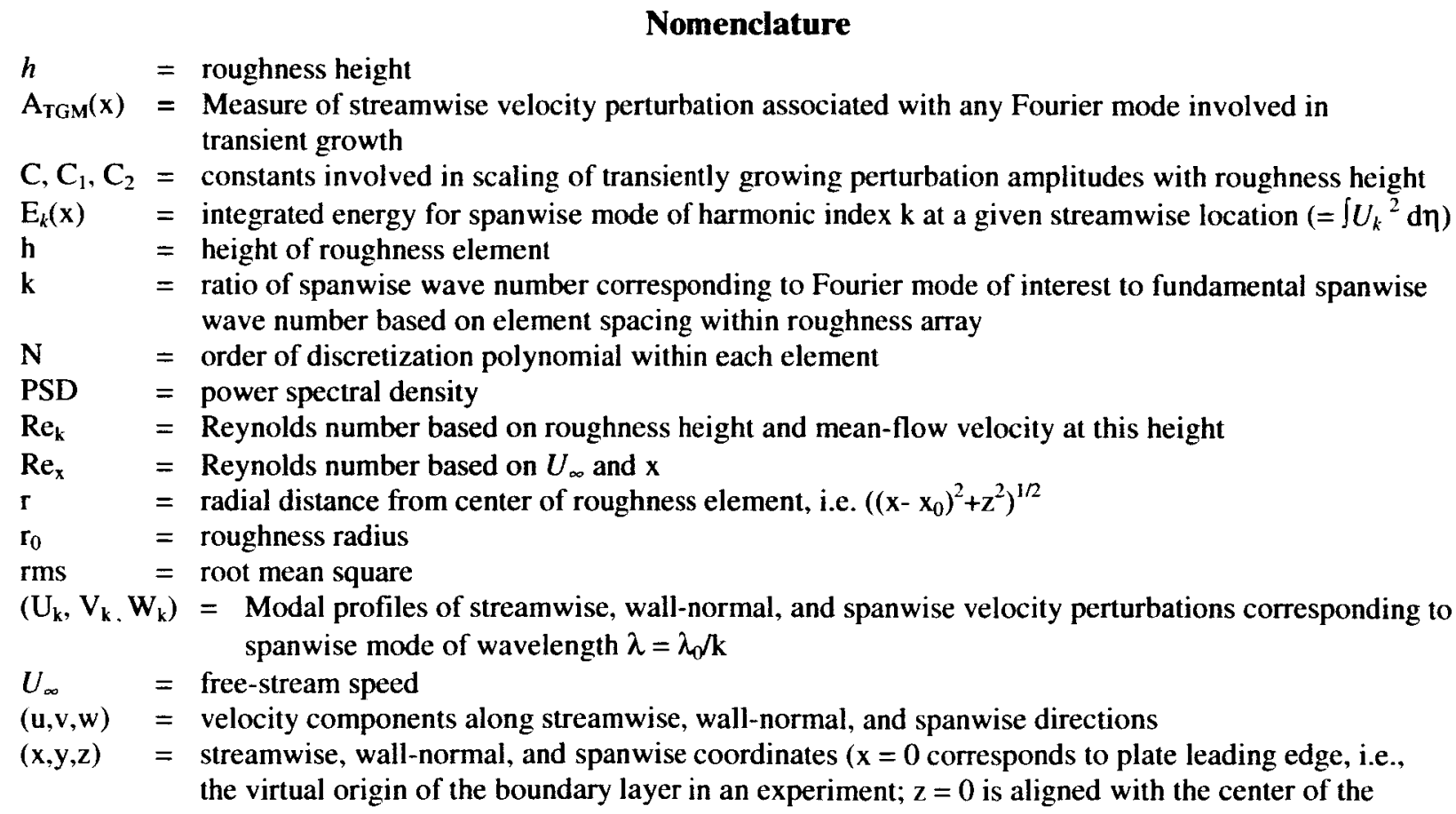

\footnotetext{
* Senior Scientist, Mathematics and Computer Science Division, 9700 S. Cass Avenue, Argonne, IL 60439.

${ }^{\dagger}$ Senior Research Scientist, Computational Modeling and Simulation Branch, M.S. 128, AIAA Associate Fellow.
} 


\begin{tabular}{|c|c|c|}
\hline & & roughness element) \\
\hline $\mathrm{x}_{0}$ & $=$ & roughness location relative to plate leading edge \\
\hline$x_{\exp }$ & $=$ & $\begin{array}{l}\text { streamwise coordinate relative to physical leading edge in the experiment of White and Ergin (2003) } \\
\left(x_{\text {exp }}=x+70 \mathrm{~mm}\right)\end{array}$ \\
\hline$\beta_{0}$ & $=$ & Wave number of the $\lambda_{0}=\lambda_{0}$ mode, non-dimensionalized by the local similarity length scale $\mathrm{x} \mathrm{Re}_{\mathrm{x}}^{-1 / 2}$ \\
\hline$\eta$ & $=$ & Blasius similarity variable $\left(\mathrm{y} / \mathrm{x} \operatorname{Re}_{\mathrm{x}}{ }^{1 / 2}\right)$ \\
\hline$\lambda$ & $=$ & spanwise wavelength of Fourier mode \\
\hline$\lambda$ & $=$ & spacing between adjacent pair of elements from spanwise roughness array \\
\hline
\end{tabular}

\section{Introduction}

$\mathrm{T}$ HE transient growth phenomenon refers to an algebraic amplification of small-amplitude disturbances prior to an exponential decay farther downstream. Physically, transient growth arises due to the "lift-up" effect associated with streamwise vortex motions (Landahl 1980). Mathematically, transient growth is a direct consequence of non-normality of the linear stability operator (Trefethen et al. 1993). Nonnormality implies that a suitable superposition of linearly stable eigenmodes may involve significant cancellation between the associated flow perturbations. Due to streamwise variations in the underlying mean flow (i.e., nonparallel effects), the destructive interference characteristics cannot be sustained while the above set of disturbances evolves in the downstream direction. The gradual weakening of mutual cancellation results in a transient growth in perturbation amplitudes before the exponential decay of the stable eigenmodes eventually comes into play.

For suitable inflow conditions, the initial cancellation is strong enough for the transient amplification ratios to meet or even exceed the range of growth factors that is known to correlate with the onset of transition due to the exponential growth of linearly unstable eigenmodes. Consequently, the transient growth paradigm has emerged as an alternate scenario for laminar-turbulent transition, especially under subcritical (i.e., linearly stable) flow conditions (Reshotko, 2001). An upper bound on the transient growth ratios is provided by the optimal growth theory (Farrell 1988, Butler and Farrell 1992, Andersson et al. 1999, Luchini 2000, Tumin and Reshotko 2001). According to this theory, the optimal initial conditions are associated with purely wall normal and spanwise velocity perturbations resembling a spanwise array of streamwise vortices; the transient growth occurs as these initial conditions evolve into streak like motions that are dominated by perturbations in the streamwise velocity.

The physical relevance of optimal growth theory to boundary-layer transition is largely dependent on receptivity characteristics of the laminar boundary layer, in particular, whether and how such optimal (or near-optimal) initial conditions can be realized in a natural disturbance environment. Transient growth of low-frequency boundary-layer disturbances due to free-stream turbulence of weak through moderate intensity has been documented in a number of experiments (Kendall 1985, Westin et al. 1994) and also predicted using theoretical models (Andersson et al. 1999, Leib et al. 1999). The work by Leib et al. (along with the related earlier works by Choudhari (1996) and Bertolotti (1997)) illustrates the need to model the transient growth phenomenon as an inhomogeneous boundary value problem associated with a physically realizable forcing environment, rather than via the optimal growth formulation alone.

The analogous issue of whether initial conditions appropriate for near-optimal growth can also be seeded via 3D surface roughness (and, if so, of what kind) has received less attention thus far. Reshotko and Tumin (2002) recently used a linear amplitude criterion based on optimal disturbance growth to correlate with an existing database for subcritical transition on rough axisymmetric nosetips. The finding that this linear criterion was able to correlate the major trends involving the effects of roughness height and surface temperature reinforced the notion that surface roughness might also be an important catalyst for inducing transient growth phenomena. However, because the maximum roughness heights associated with the experimental database were comparable to the boundary-layer thickness, the reasons behind the success of a transition criterion based on a purely linear model are not clear at this stage.

Quantitative measurements of the stationary disturbance field induced by 3D roughness elements in the form of a circular disk were carried out by Tani et al. (1962), and then by Kendall (1982) in conjunction with his pioneering measurements of the transient growth due to weak free-stream turbulence. Due to the difficulty in measuring the weak velocity perturbations induced by an isolated roughness element, Kendall had to use signal-averaging 
techniques to enhance the accuracy of the measured data. Gaster et al. (1994) circumvented these challengcs by using a surface diaphragm oscillating at very low frequency. Like Kendall, Gaster et al. observed a switchover in the spanwise disturbance profile from a unimodal behavior immediately behind the roughness element to a profile involving multiple spanwise extrema at farther downstream locations. However, the respective datasets are found to differ with respect to the number and the signs of these spanwise peaks.

Gaster et al. noted that the appearance of additional spanwise peaks was accompanied by a significant transient streamwise growth within a band of low wave number disturbances (compared with the peak wave number of the unimodal disturbance profile). The transient growth phenomenon was also captured in the direct numerical simulations by Joslin and Grosch (1995), who modeled the low-frequency oscillation of the diaphragm from Gaster et al. as a stationary hump. However, an uncertainty in the mode shape of the vibrating diaphragm in the experiment prevented an in-depth quantitative comparison with the measured data.

The investigations by Kendall (1982), Gaster et al. (1994), and Joslin and Grosch (1995) were carried out prior to the development of a spatial theory for optimal growth (Andersson et al. 1999, Luchini 2000, Tumin and Reshotko 2001). More recent wind tunnel experiments by White (2002) and White and Reshotko (2002) also demonstrated the occurrence of roughness-induced transient growth in the intermediate wake region for a variety of roughness configurations involving cylindrical (i.e., circular disk) roughness elements and sand-grain roughness. Although the measurements of White (2002) were consistent with some aspects of the optimal growth theory, the magnitude of the transient growth associated with a spanwise periodic array of cylindrical elements was found to be suboptimal. In reducing the hot wire data to determine the spanwise variation in the mean velocity, White (2002) used a linear extrapolation technique to estimate the local plate surface location at a given spanwise coordinate. Subsequent analysis by White and Ergin (2003) showed that the distorted mean-flow profile near the wall has a finite curvature within the region of transient growth and, therefore, some of the results reported in White (2002) may not have been correct. White and Ergin presented additional data for a spanwise array of cylindrical roughness elements that was reduced by using a spanwise global method for estimating the plate surface location. These new measurements further quantified the variation in the amplitude of algebraically growing disturbances with respect to the roughness height.

Tumin and Reshotko (2004) have presented a clever theoretical analysis of roughness-induced transient growth; they used a generalization of the method that was developed and applied earlier for receptivity predictions in the context of linearly unstable Tollmien-Schlichting modes. Using biorthogonal eigenfunctions of the linear, quasiparallel disturbance equations, they were able to predict the stationary disturbances in the wake of an array of roughness elements without having to solve for the disturbance field either above or upstream of the roughness array. The disturbance profiles obtained with this procedure were used as initial conditions for a marching procedure to confirm the realizability of optimal growth for this configuration. Even though the flow conditions used in their case study were nominally identical to those of the White and Ergin (2003) experiment, Tumin and Reshotko's results show some differences in the characteristics of the observed transient growth. It is not clear whether or to what degree those differences may be attributed to the differences in planform shape (namely, square roughness elements in the analysis versus circular ones in the experiment) or the departure in experiment from theoretical assumptions (linearity, and parallel and quasi-parallel flow assumptions in the nearfield and farfield, respectively).

In summary, the qualitative features of roughness-induced transient growth are somewhat consistent between theory and experiments. However, additional theoretical studies are necessary to establish quantitative agreement for a common set of flow and roughness parameters. Such studies are also desirable in view of the various challenges in making reliable measurements of small-to-moderate amplitude spanwise variations in the mean velocity field, so as to help resolve some of the discrepancies between previously reported experiments involving roughness-induced disturbances. To help address these issues, this paper presents numerical simulations for a select set of test conditions from the experiments of White and Ergin (2003). A secondary objective behind this effort is to extend the significant body of previous numerical investigations from roughness elements with a smooth shape (i.e., $\mathrm{C}^{0}$ and smoother) to the cylindrical, disk-shaped roughness elements that are encountered in a number of research studies as well as technological applications.

An outline of the paper is as follows. In section II, we outline the flow configuration and the numerical algorithm used for the simulations. Section III describes the results obtained for the baseline configuration, with an 
emphasis on detailed comparison with the White and Ergin (2003) data as well as on results pertaining to flow quantities that cannot be easily measured in an experiment. Findings from a rather limited parameter study involving additional roughness configurations are outlined in section IV. Concluding remarks are presented in section V.

\section{Flow Configuration and Computational Procedure}

The primary flow configuration used for the numerical simulations corresponds to a flat-plate boundary layer with a spanwise periodic array of circular-disk roughness elements located at the distance of $x_{0}=230 \mathrm{~mm}$ from the sharp leading edge. In comparison with free-stream turbulence, which provides sustained external forcing throughout the streamwise extent of the flow, a localized roughness array is perhaps more relevant to the initial value problem modeled by the optimal growth theory. The diameter of the roughness elements is equal to $6.35 \mathrm{~mm}$, with the spacing between each pair of adjacent elements being $\lambda_{0}=19 \mathrm{~mm}$, i.e., approximately 3 times the roughness diameter. The free-stream speed is $10 \mathrm{~m} / \mathrm{s}$. The baseline simulations correspond to a roughness height of $h=0.57 \mathrm{~mm}$ (i.e., $\mathrm{Re}_{\mathrm{k}}=119$ ). These values correspond to case 3 in the experiments of White and Ergin (2003), which was the focus of most of the results presented in their paper.

The Nek5000 code developed at the Argonne National Laboratory (Fischer et al. 2002) was used for the numerical simulations. In this code, the unsteady incompressible Navier-Stokes equations are integrated in time by using the spectral element discretization in space and a third-order, operator-splitting formulation in time. The time advancement scheme includes an implicit, linear Stokes solver combined with an explicit subintegration of the nonlinear advection term. The discretized Stokes problem is solved by using operator splitting, which results in decoupled equations for the velocity components and pressure. Despite the fact that only $\mathrm{C}^{0}$ continuity is imposed across element interfaces, the overall results are known to be spectrally accurate in space. The combination of geometric flexibility and spectral accuracy makes the spectral element method particularly well-suited for simulating the present flow configuration, as well as to meet the long-term goal of transition simulations involving complex geometry such as multielement, high-lift configurations.

To facilitate a series of simulations for multiple roughness heights, the computational domain is split into a nearfield region including the roughness array and a farfield region occupying the smooth downstream section. The nearfield subdomain is discretized via an unstructured array of hexahedral elements, some of which have curved boundary faces, while the farfield domain is composed of a regular array of hexahedral elements that admits the use of fast tensor-product solvers. The interface between the nearfield and farfield zones is a conforming one, so that the adjacent elements on opposite sides of the interface align perfectly with each other. Figure 1 shows a schematic of the grid topology, including a close-up of the grid in the vicinity of the roughness array. The nearfield and farfield portions of the grid contain a minimum of 762 and 2160 elements, respectively. The nearfield domain in the baseline case extends from $19 r_{0}$ upstream of the roughness location to $15 r_{0}$ in the downstream direction, whereas its free-stream boundary is located at $20 \mathrm{r}_{0}$, i.e., nearly 21 times the boundary-layer thickness at the roughness location. The farfield domain begins at $8 \mathrm{~mm}\left(\approx 2.52 \mathrm{r}_{0}\right)$ downstream of the roughness location $\left(\mathrm{x}_{0}\right)$, and extends up to $\mathrm{x} \approx \mathrm{x}_{0}$ $+500 \mathrm{~mm}$. Progressive stretching of the wall-normal grid with increasing $\mathrm{x}$ provides approximately constant resolution across the boundary layer at all streamwise locations. The streamwise grid is stretched in the downstream direction, because the streamwise resolution requirements for streak like motions are less stringent than those for the nearfield solution.

The roughness heights employed in the present set of simulations are small enough that the induced disturbance field is purely stationary. Accordingly, the simulations are advanced in time until the last pair of solution snapshots separated by a finite time interval can be considered as identical within a specified tolerance. Preliminary simulations for the baseline case used a full wavelength domain $\left(-\lambda_{0} / 2 \leq z \leq \lambda_{0} / 2\right)$, with periodic boundary conditions along the spanwise direction. The results of these simulations confirmed the spanwise symmetry of the disturbance field, in agreement with the experimental observations of White and Ergin (2003). Accordingly, all of the simulations reported in this paper have been carried out by using a computational domain with a spanwise extent of $-\lambda_{0} / 2 \leq \mathrm{z} \leq 0$ and symmetry conditions at both ends.

The numerical simulations are initialized with the Blasius similarity solution, shifted appropriately in the wallnormal direction in the region of the roughness array. The relevant Blasius profiles are also imposed along the inflow boundary, whereas no-slip conditions are used along the entire plate surface. The free-stream boundary 
conditions enforce an irrotational disturbance behavior near the farfield boundary, and a convective boundary condition was imposed along the downstream (i.e., outflow) boundary. The robustness and effectiveness of the various boundary conditions were verified in separate computations involving spatial simulations of instability wave amplification within a parallel boundary-layer flow (Fig. 2).

For spectral element calculations, grid convergence can be assessed by reducing the size of the elements and/or by increasing the order $\mathbf{N}$ of the discretization polynomial within each element. Typically, the latter approach is more convenient; this approach was adopted in the present study to evaluate the grid convergence of the computed disturbances. In particular, nearfield and farfield solutions obtained with 11th order and 7 th order elements, respectively, were compared with each other for the case of $h=0.665 \mathrm{~mm}$ (i.e., the largest roughness height considered in the present study). Figures $3 \mathrm{a}$ and $3 \mathrm{~b}$ illustrate the agreement between streamwise and spanwise velocity components sampled from the $\mathrm{N}=7$ and $\mathrm{N}=11$ solutions, respectively, at an $\mathrm{x}$-location near the outflow of the nearfield domain. For the most part, a similar agreement was found between the corresponding solutions within the farfield domain, except for a noticeable discrepancy near the outflow of the domain. However, the magnitude of this discrepancy was small and was confined to the far downstream region wherein the streamwise element size had become rather large. Comparison between the nearfield and farfield solutions within the domain of overlap (wherein the two domains involve somewhat different grid distributions) provided an additional degree of check with respect to the grid convergence of the computed solution, in addition to validating the domain decomposition approach used in these simulations.

Nearly all of the postprocessing of the simulation data was based on interpolating the computed solution to a specified output grid involving uniform spacing of points along the spanwise direction. To minimize any loss of accuracy, spectral interpolation based on the same degree of polynomial as that used for the simulation itself was employed during this interpolation. Fast cosine and sine transforms were used to decompose the interpolated solution into Fourier modes; integrated modal energies at the streamwise locations of interest were evaluated using the trapezoidal rule for quadrature.

\section{Results for Baseline Configuration}

Despite the relative simplicity of the overall problem, the parametric space involved is rather large, including both mean boundary-layer parameters at the roughness location $\left(\operatorname{Re}_{\theta}\right.$, shape factor $\mathrm{H}$, pressure gradient parameter $\beta$, etc.) and relevant attributes of the roughness geometry (shape, planform size and aspect ratio, and roughness height, i.e. $\mathrm{Re}_{\mathrm{k}}$ ). Limitations of computing resources precluded a detailed parametric study of roughness-induced transient growth. Thus, a limited case study has been performed to validate simulation results against measured data, to provide additional data for validating simpler theoretical models such as that of Tumin and Reshotko (2004), and to delineate the influence of roughness geometry on the process of transient growth. The idea behind the case study was that if simplified theoretical models can be sufficiently improved and validated via the combination of wind tunnel experiments and numerical simulations, then those same models can be easily used for a detailed parametric study of roughness-induced transient growth. With that in mind, simulation results for the baseline configuration of $\mathrm{h}=0.57 \mathrm{~mm}$ are presented in this section; results for the additional roughness configurations are deferred until section IV. Unless stated otherwise, all of the simulation results presented in sections III and IV are based on $\mathrm{N}=$ 11.

\section{A. Overall Features}

The salient features of the near-wall flow field in the vicinity of the cylindrical roughness element may be gleaned from Fig. 4. The bottom half of the figure displays the surface pressure contours, and the upper half depicts the distribution of streamwise velocity component at a small, fixed height just above the surface. By confining the range of streamwise velocity contours to negative values, the top half of the figure clearly highlights the two separate regions of flow reversal-a primary region behind the roughness element and a smaller one near the centerline just in front of the element. The associated pattern of 2D streamlines within this plane is also shown to emphasize the direction of the in-plane velocity vectors.

The surface pressure distribution in the bottom half of Fig. 4 is partially analogous to that predicted by Smith et al. (1977) by applying the linearized version of triple-deck theory to an isolated roughness element with a smoother (i.e., $\mathrm{C}^{1}$ ) height distribution. In both their theoretical results and the present simulation, one observes peak pressures along the centerline just ahead of the roughness element, lowest pressure above the roughness itself, and a pressure 
recovery in the wake region. However, the present simulations suggest the pressure recovery to be virtually monotonic, i.e., without the small overshoot just behind the roughness element as predicted by the linearized theory. The discrepancy between the theoretical predictions of Smith et al. (1977) (as well as Tumin and Reshotko 2004) and the simulation results herein may be due to of the following: the differences in roughness element shape and spanwise spacing and the effects of nonlinearity which had been neglected in the theory (and which may alter the effective roughness shape when the region of flow reversal behind the circular roughness has been taken into account). Additional calculations are necessary to help identify the precise cause behind this discrepancy.

The computed distribution of streamwise shear stress within the wake region confirmed the theoretically predicted feature of a streamwise elongated "corridor" involving a pair of high-speed streaks, one on each side of the centerline (see Fig. 21 from Tumin and Reshotko 2004). The centerline region displays a low-speed streak involving a progressively reducing velocity deficit. Visualization of the 3D flow field suggests that the occurrence of high-speed streaks may be attributed to the horseshoe vortex system formed as the spanwise vorticity lines in the incoming flow wrap around the cylindrical roughness element in the near-wall region. Velocity contours in the wake of the roughness array (Fig. 5) illustrate the longevity of the vortex structures associated with the high-speed streaks. The spanwise structure of the wake disturbances is qualitatively similar to the computations of Joslin and Grosch (1995) and Woerner et al. (2004) for smoother roughness shapes.

The streamwise velocity contours at a couple of selected streamwise stations downstream of the roughness array are shown in Figs. 6(a) and 6(b). The locations of these stations were chosen to allow a direct comparison with Figs. 4 and 7, respectively, from White and Ergin (2003). The near-wake contours at $x-x_{0}=10 \mathrm{~mm}$ (Fig. 6(a)) indicate a single dominant low-speed streak near the centerline, similar to that observed in the experiments of Kendall (1982), Gaster et al. (1994), and White and Ergin (2003). The high-speed streaks mentioned previously emerge farther downstream, as seen from Fig. 6(b).

Streamwise evolution of the total disturbance energy (i.e., mean square amplitudes integrated across the profile at a fixed streamwise location) illustrates the weak decay in spanwise varying perturbations within the far-wake region as shown by the solid curve in Fig. 7. To maintain consistency with the data reported by White and Ergin (2003), the disturbance energy profiles are integrated with respect to the local similarity variable at each streamwise station. This choice of wall-normal length scale masks the effects of boundary-layer growth in the streamwise direction. However, the energy of the spanwise varying perturbations is found to decay (albeit more weakly) even after the boundary-layer growth has been accounted for in calculating the total disturbance energy at any given location. The dashed curve in Fig. 7 is based on a reference state corresponding to the $z=-\lambda_{0} / 2$ plane, which lies in between an adjacent pair of roughness elements. All of the features of the disturbance energy evolution in Fig. 7 are in satisfactory agreement with Fig. 11 from White and Ergin (2003).

\section{B. Transient Growth and Comparison with Measured Data}

Having validated the general features of the computed disturbance field against the measured data, we next examine the downstream evolution of the individual Fourier harmonics in order to quantify detailed features of the transient growth induced by the baseline roughness array with $\mathrm{h}=0.57 \mathrm{~mm}$. The disturbance profiles at the fundamental spanwise wave number and its first three harmonics at streamwise locations of $x-x_{0}=10 \mathrm{~mm}$ and $x$ $\mathrm{x}_{0}=80 \mathrm{~mm}$ are shown in Figs. 8(a) and 8(b), respectively. These two locations are the same as those considered in Figs. 6(a) and 6(b) discussed previously. A comparison of the modal amplitudes from Figs. 8(a) and 8(b) indicates the switchover in the dominant Fourier component from $\lambda=\lambda_{0}$ at the upstream location to the $\lambda=\lambda_{0} / 3$ mode farther downstream, as previously noted by White and Ergin (2003). The root mean square (rms) disturbance amplitudes at both locations agree quite well with the measured data presented in Figs. 6 and 9, respectively, from White and Ergin. A scrutiny of the rms profiles at other stations did not reveal any double peaks, supporting the claim by White and Ergin that the presence of double peaks in their earlier results (White 2002) was caused by inaccuracies related to the wall finding algorithm.

The modal profiles from White and Ergin (2003) correspond to the power spectral density (PSD) at a given spanwise wavelength rather than to the mean-square amplitude over each spectral peak as plotted in Fig. 8 . Assuming a constant shape and wave number bandwidth across the relevant set of peaks in the measured PSD data, the results from Fig. 8 can be compared with the modal profiles in Figs. 6 and 9 of White and Ergin via a simple rescaling. Accordingly, we have rescaled the computed mean-square amplitudes in Fig. 8 by a constant factor of 13.7. This particular scaling factor was chosen to provide an approximate match between the respective peaks in the

6

American Institute of Aeronautics and Astronautics 
modal profiles for $\lambda=\lambda_{0}$ at $\mathrm{x}-\mathrm{x}_{0}=10 \mathrm{~mm}$. Reasonable agreement is obtained between simulation results and the measured data, both in regard to the mode shapes and amplitudes for $\lambda=\lambda_{0}, \lambda=\lambda_{0} / 3$, and $\lambda=\lambda_{0} / 4$. A noteworthy discrepancy involves what appears to be a greater reduction in the fundamental mode amplitude in the experiment as the measurement location is moved from $x-x_{0}=10 \mathrm{~mm}$ to $x-x_{0}=80 \mathrm{~mm}$. As we observe later, the fundamental mode does not decay monotonically between the two locations, which may account for the discrepancy related to the decay factor. A comparison between Figs. 8(a) and 8(b) also confirms the outward movement in the peak locations corresponding to the modal profiles for $\lambda=\lambda_{0} / 3$ and $\lambda=\lambda_{0} / 4$ as one moves downstream from $\mathrm{x}-\mathrm{x}_{0}=10 \mathrm{~mm}$ to $\mathrm{x}-$ $\mathrm{x}_{0}=80 \mathrm{~mm}$.

Streamwise evolution of the integrated modal energy for the wave number components $\lambda=\lambda_{0}, \lambda=\lambda_{0} / 3$, and $\lambda=$ $\lambda_{0} / 4$ is shown in Fig. 9(a). All three modes exhibit transient growth before decaying farther downstream; however, the fundamental mode undergoes a rapid and substantial decay before the beginning of its algebraic growth (and its eventual decay is not captured within the computational domain). The shorter wavelength modes, on the other hand, begin to amplify almost immediately in the wake of the array, similar to the experiment. The $\lambda=\lambda_{0} / 4$ mode reaches its peak amplitude before the other modes, and is closely followed by the $\lambda=\lambda_{0} / 3$ mode which dominates the disturbance amplitudes in the intermediate wake region (up to approximately $170 \mathrm{~mm}$ downstream of the roughness array). Interestingly enough, the minimum of the fundamental mode energy occurs just slightly upstream of the maximum in the $\lambda=\lambda_{0} / 3$ mode on the scale of this plot. Again, similar to Fig. 7 , we have used the local similarity length scale to nondimensionalize the wall-normal coordinate $\eta$ in order to permit a visual comparison with the measurements of White and Ergin which have been reproduced in Fig. 9(b) for the reader's convenience. (Note that the comparison may be only qualitative at this stage because of the potentially nontrivial conversion from PSD to squares of the modal amplitudes.) With the choice of a streamwise varying length scale, the energy metric $\left(E_{1}(x)\right)$ for the fundamental mode appears to reach a plateau near $x_{\text {exp }}=600 \mathrm{~mm}$ (i.e., $x-x_{0}=300 \mathrm{~mm}$ ) even though the actual disturbance energy continues to grow throughout the streamwise region included in Fig. 9(a). The continued growth of the fundamental mode appears consistent with the predictions of the optimal growth theory (Andersson et al. 1999, Tumin and Reshotko 2001), even though the initial locations considered in those calculations were typically much closer to the leading edge than the roughness array considered herein. If the previously published optimal growth results were to hold even for the present case, then the fundamental mode $\lambda=\lambda_{0}$ has the potential to amplify until the local spanwise wave number has reached a value of $\beta_{0} \approx 0.45$, i.e., until approximately $x=1175$ $\mathrm{mm}$. To determine whether such optimal growth is realized in this case, the present far-field calculations will have to be extended upto larger values of $x$. The additional far-field calculations would also help identify (as well as resolve) any connection between a possible lack of grid convergence in the far-downstream portion of the present farfield domain and the earlier crossover in the computation between the modal energies for $\lambda=\lambda_{0}$ and $\lambda=\lambda_{0} / 3$ as the latter mode decays across this region.

Tumin and Reshotko (2004) made the key observation that a sign reversal of the $U_{k}$ modal profiles in the wake of the roughness array creates conditions that locally resemble the theoretically predicted optimal initial profiles corresponding to a spanwise array of streamwise vortices. Conventional applications of the optimal growth theory consider a streamwise interval that begins at the leading edge (or very close to it), in which case these streamwise vortices actually protrude outside of the boundary layer. Tumin and Reshotko presented an optimal growth calculation based on a streamwise interval beginning at a finite distance downstream of the leading edge; this example case suggests that the optimal initial conditions for transient growth over regions away from the leading edge also involve a vortical secondary flow, which is now embedded inside the finite-thickness boundary layer at the starting location. The evolution of the modal profiles in Fig. 10 shows that the streamwise velocity perturbations at $\lambda=\lambda_{0}$ indeed undergo a sign change near $x-x_{0}=35 \mathrm{~mm}$ (i.e., $x_{\exp }=335 \mathrm{~mm}$ ), which corresponds to the minimum in fundamental mode energy in Fig. 9(a). The secondary flow at this location involves the modal profiles shown in Fig. 10(b) and 10(c), which are analogous in shape to those predicted by Tumin and Reshotko.

Because of the small magnitudes of the wall-normal and spanwise velocity perturbations, it is difficult to measure those perturbations in a wind tunnel experiment. However, that difficulty is less severe in the numerical simulations; we find that the "initial" disturbance energy based on just the $v$ ' and $w$ ' perturbations at $\lambda=\lambda_{0}$ is given by $\int\left(V_{1}^{2}(\eta)+W_{1}^{2}(\eta)\right) d \eta \approx 5.76 \times 10^{-6}$ at $x-x_{0}=36 \mathrm{~mm}$. This translates into an algebraic growth in the modal energy $E_{1}(x)$ by a factor of nearly 191 over the transient growth interval $x_{\exp }=336 \mathrm{~mm}$ to $x_{\exp }=650 \mathrm{~mm}$ included in the computational domain. Of course, from a physical standpoint, one must keep in mind that the above growth is preceded by an even larger disturbance decay upstream of the minimum in $E_{1}(x)$; hence, the overall significance of 
transient growth via a spanwise periodic roughness array cannot be determined at this stage. Indeed, a roughness array with subcritical heights can influence the transition process in multiple ways. It can modify the stability characteristics via both strong but localized disturbances in the vicinity of the roughness array and weaker but persistent disturbances in the transient growth region (see Cossu and Brandt (2002) for a theoretical study of the latter mechanism). Furthermore, it is well-known that the roughness array will also serve as a catalyst for the generation of both 2D and 3D Tollmien-Schlichting instability waves, which may or may not begin to grow until much later depending on the combination of roughness location and spacing and the frequencies of unsteady freestream disturbances.

\section{Influence of Roughness Height, Diameter, and Shape on Transient Growth}

Additional simulations were performed to discern the effects of roughness geometry on the transient growth phenomenon. A brief outline of the pertinent findings from these simulations is provided in this section.

\section{A. Effect of Roughness Height}

According to White and Ergin's data (2003), both the transient growth characteristics and the subsequent exponential decay of the $\lambda=\lambda_{0} / 3$ mode are approximately uniform across the range of roughness heights considered in their measurements. Yet, the modal amplitude at any fixed station downstream of the roughness array is a nonlinear function of the roughness height parameter. Both of these findings are qualitatively consistent with previous theoretical development for the closely related problem of roughness-induced excitation of inviscid, stationary vortex instabilities in a boundary layer (Choudhari and Duck, 1996). The asymptotic theory showed that the nearfield dynamics for the roughness elements under consideration becomes nonlinear at relatively small roughness heights compared with the boundary layer thickness, but that the disturbance dynamics farther downstream of the roughness elements remains linear to the leading order (until, of course, the exponential growth of those inviscid instabilities causes nonlinear effects to come into play once again). In other words, the influence of nonlinearity is confined to the output of the receptivity process, i.e., to determining the scaling of the effective initial amplitude as a function of the roughness height, and that the initial growth of the generated vortex/streak mode is independent of the roughness height parameter to the leading order.

White and Ergin were able to correlate their data for the transient growth of $\lambda=\lambda_{0} / 3$ and $\lambda=\lambda_{0} / 4$ modes with a quadratic fit of the form $E_{3}, E_{4} \propto \operatorname{Re}_{k}{ }^{2}$ at any fixed $x$. To assess this scaling behavior within the computational framework, additional simulations were carried out for $h=0.665 \mathrm{~mm}$ (i.e., $\mathrm{Re}_{\mathrm{k}} \approx 162$ ). A comparison of the nearfield solutions for $\mathrm{Re}_{\mathrm{k}} \approx 162$ and the baseline case $\left(\mathrm{Re}_{\mathrm{k}} \approx 119\right)$ presented in section III showed that the $16.7 \%$ increase in roughness height resulted in approximately $24 \%$ percent increase in the length of the reversed flow region along $z=0$ behind the roughness element. Modal analysis of the wake disturbances (Fig. 11) suggests that the $\mathrm{Re}_{\mathrm{k}}{ }^{2}$ scaling applies to modal energies of all four modes under consideration (i.e., $\lambda=\lambda_{0}, \lambda_{0} / 2, \lambda_{0} / 3$, and $\lambda_{0} / 4$ ).

White and Ergin (2003) did not provide any rationale for choosing the particular scaling noted above. The $\operatorname{Re}_{\mathrm{k}}{ }^{2}$ scaling for the modal energy translates into an amplitude scaling of the form $A_{T G M} \sim C e_{k}$, where $A_{T G M}$ is a measure of the streamwise velocity perturbation associated with a given mode undergoing transient growth (e.g., $\mathrm{A}_{\mathrm{TGM}}$ could be interpreted as $\max \left(\left|U_{k}(\eta)\right|\right)$ within the region of transient growth). This amplitude scaling is equivalent to $A_{T G M} \sim C_{2} h^{2}$ in view of the approximately linear behavior of the Blasius profile over the range of roughness heights included in the correlation. Of course, the purely quadratic scaling for the perturbation amplitudes cannot hold at sufficiently small roughness heights; therefore, the truncated form of regular perturbation expansion, $A_{\mathrm{TGM}} \sim \mathrm{C}_{1} \mathrm{~h}+\mathrm{C}_{2} \mathrm{~h}^{2}$, must be considered in general. Numerical simulations are well-suited to characterize the linear behavior at small heights (wherein the experimental measurements might encounter difficulties in maintaining an adequate signal-to-noise ratio because of the reduced perturbation amplitudes) as well as to help delineate the transition from a linear behavior to the quadratic dependence observed in experiments and simulations when the $\mathrm{Re}_{\mathrm{k}}$ parameter is sufficiently large. The parameter study required for this characterization is currently under way.

\section{B. Effect of Ratio of Roughness Diameter to Array Spacing}

To help understand the factors responsible for the absence of transient growth in the $\lambda=\lambda_{0} / 2$ mode, we carried out an additional simulation for the larger roughness radius of $r_{0}=\lambda_{0} / 4$ (i.e., roughness diameter of one-half the array spacing). To avoid the complexity of the split domain calculation, the simulation for the larger planform-size roughness array was based on a single structured grid of elements within a rectangular box volume. Also, the no- 
slip conditions over the roughness array were transferred to the plate surface $y=0$ by using a perturbation expansion based on a linear Taylor series expansion in the roughness height parameter. Since the solver is based on the full Navier-Stokes equations, the height of the roughness element was reduced to $h=0.12 \mathrm{~mm}$ in order to reduce the influence of the nonlinear terms yet avoid a loss of accuracy in analyzing the perturbations. A half-wavelength cosine fairing was used to round off the edges of the cylindrical roughness element over the fringe region $\mathrm{r} / \mathrm{r}_{0}=1 \pm$ 0.25 . Based on the grid convergence study for the baseline configuration, the discretization order was also reduced from $\mathrm{N}=11$ to $\mathrm{N}=8$ for this configuration; however, no additional grid convergence checks were performed.

Because the spacing between the high-speed streaks scales with the roughness diameter, the $\lambda=\lambda_{0} / 2$ mode emerges in this case as the dominant Fourier harmonic in a large portion of the wake region. However, similar to the baseline configuration, this mode decays monotonically, without any evidence of transient growth. The $\lambda=\lambda_{0}$ and $\lambda=\lambda_{0} / 3$ modes do show an algebraic amplification; however, their amplitudes are significantly weaker than the baseline configuration $\left(r_{0}=\lambda_{0} / 6\right)$. Furthermore, the growth of the fundamental mode begins relatively farther downstream, and (unlike the baseline configuration) the $\lambda=\lambda_{0} / 4$ mode does not grow at all. These findings indicate the significance of roughness diameter (in relation to array spacing) as a significant parameter in determining the quantitative aspects of roughness-induced transient growth.

\section{Roughness Elements with Square Planform and Comparison with Theory of Tumin and Reshotko (2004)}

The theoretical predictions of Tumin and Reshotko (2004) based on a linear model are in qualitative agreement with the measurements of White and Ergin (2003) and the simulation results despite the obviously nonlinear regime of roughness heights in both experiment and simulations. However, there is a notable discrepancy between theory and measured data with regard to the onset of transient growth in the $\lambda=\lambda_{0} / 3$ and $\lambda=\lambda_{0} / 4$ modes. The algebraic growth of these modes begins almost immediately behind the roughness array in the experiment, whereas it is postponed until approximately $150 \mathrm{~mm}$ downstream of the roughness array according to the theoretical predictions. To help ascertain whether the discrepancy is related to differences in planform shapes of the roughness elements (a square shape in the calculations of Tumin and Reshotko, versus circular disks in the experiment), an additional simulation was carried out for square shaped elements with the same width as the roughness diameter in the baseline configuration (i.e., $r_{0}=\lambda_{0} / 6$ ). In an attempt to simulate the linear behavior modeled by theory, the modified simulation procedure based on a transfer of roughness boundary condition to the plate surface, as described in section IV-B, was also applied to this particular configuration. The results indicated that the transient growth features for square elements were analogous to those for the circular elements of comparable size, in that the $\lambda=$ $\lambda_{0} / 3$ and $\lambda=\lambda_{0} / 4$ modes again began to amplify just downstream of the roughness array. Additional work is therefore necessary to identify the cause behind this discrepancy. However, once the theoretical model has been validated (and refined if necessary) for a simple configuration of this type, such models could be extended to the difficult case of distributed, sand-grain roughness (which will require rather prohibitive resources for numerical simulations) as well as being used as a predictive tool for roughness-induced transient growth.

\section{Concluding Remarks}

Direct numerical simulations have been carried out to shed further light on the phenomenon of roughnessinduced transient growth induced by a spanwise periodic array of roughness elements. For roughness arrays with $\mathrm{Re}_{\mathrm{k}} \approx 119$ and $\mathrm{Re}_{\mathrm{k}} \approx 162$, the computed features of transient growth are consistent with those measured in the experiment of White and Ergin (2003), including the nonlinear scaling of perturbation energy with the roughness height parameter. The agreement between simulation results and the measured data also confirms the accuracy of the spanwise-global wall-finding algorithm used by White and Ergin (2003) over the local wall-finding procedure used previously by White (2002). Good quantitative agreement has been shown between the computed and the measured rms disturbance amplitudes for the baseline configuration of $\mathrm{Re}_{\mathrm{k}} \approx 119$. The agreement pertaining to modal amplitudes is somewhat less satisfactory, and additional work is required to help identify the factors underlying this discrepancy. Similarities between the transient growth due to roughness array composed of square shaped and circular roughness elements indicate the likely significance of roughness size relative to array spacing as a primary control variable, at least for the class of cylindrical roughness elements examined herein.

A number of relevant issues still need to be addressed, such as in-depth comparison with both experiments and linearized theory, calculations for additional roughness heights so as to better quantify the overall scaling of disturbance amplitudes (and, secondarily, the magnitudes of transient growth) with $\mathrm{Re}_{\mathrm{k}}$. Further calculations would 
also help delineate the critical range of roughness heights beyond which the flow becomes unsteady and vortex shedding occurs as the precursor to the formation of turbulent spots (Acarlar and Smith 1987, Klebanoff et al. 1992). An additional, relatively straightforward extension of the present simulations would involve the roles of planform aspect ratio (i.e. elliptical instead of circular disk roughness elements) and spanwise asymmetry of the planform shape of roughness elements.

While the work provides an independent confirmation for the occurrence of transient growth due to surface roughness, it also underscores the weakness of the peak disturbance amplitudes involved in the transient growth process. Although it seems unlikely that the role of nearfield disturbances can be diminished via proper selection of roughness geometry, investigations focused on the identification of optimal roughness distributions that maximize the amplitude of the wake disturbances while keeping the amplitudes of the nearfield disturbances to a minimum would also be beneficial.

Finally, a number of recent studies (Bakchinov et al. 1995, Asai et al. 2002, Cossu and Brandt 2002, Wu and Luo 2003) have addressed the influence of stationary streaks and optimal growth disturbances on the stability of the unperturbed boundary layer and/or the interaction between stationary streaks and the unsteady Tollmien-Schlichting instabilities in the boundary layer. Additional studies of this type in the specific context of roughness-induced transient growth would be helpful, especially from the standpoint of placing the roughness-induced transient growth in a correct physical perspective.

\section{Acknowledgments}

The authors thank Prof. Edward White and Prof. Eli Reshotko for discussions of the experimental work at Case Western Reserve University. Technical communications with Prof. Anatoli Tumin from the University of Arizona and Dr. Craig Street from NASA Langley Research Center are also gratefully acknowledged. A portion of this research was carried out while MC was visiting the Imperial College of Science of Technology, London, U.K. He would like to thank Dr. Xuesong Wu and Prof. Philip Hall for their kind hospitality during this visit and the EPSRC for making this visit possible via Grant GR/S59635.

\section{References}

Acarlar, M.S., and Smith, C.R., "A Study of Hairpin Vortices in a Laminar Boundary Layer. Part 1." J. Fluid Mech., Vol. 175, pp. $1-42,1987$.

Andersson, P., Berggren, M., and Henningson, D.S.. "Optimal Disturbances and Bypass Transition in Boundary Layers," Phys. Fluids, Vol. 11(1), pp. 134-150, 1999.

Asai, M., Minagwa. M., and Nishioka, M., "The Instability and Breakdown of a Near-Wall Low-Speed Streak," J. Fluid Mech., Vol. 455, pp. 289-314, 2002.

Bakchinov, A.A., Grek, G.R., Klingmann. B.G.B., and Kozlov, V.V., "Transition Experiments in a Boundary Layer with Embedded Streamwise Vortices," Phys. Fluids, Vol 7, pp. 820-832, 1995.

Bassom, A., and Hall, P., "The Receptivity Problem for O(1) Wavelength Gortler Vortices," Proc. Roy. Soc. London Ser. A., Vol. 446, pp.499-516, 1994.

Bassom, A., and Seddougui, S., "Receptivity Mechanisms for Gortler Vortex Modes," Theor. Comp. Fluid Dyn., Vol. 7, pp.317-339, 1995.

Bertolotti, F., "Response of the Blasius Boundary Layer to Free-Stream Vorticity,"Phys. Fluids, Vol 9(8), pp. 2286-2299, 1997.

Butler, K.M., and Farrell, B.F., "Three-Dimensional Optimal Perturbations in Viscous shear Flow," Phys. Fluids A, Vol 4(8), pp. 1637-1650, 1992.

Choudhari, M., "Boundary-Layer Receptivity to Three-Dimensional Unsteady Vortical Disturbances in Free Stream," AIAA Paper 96-0181, 1996.

Choudhari, M., and Duck, P.W., "Nonlinear Excitation of Inviscid Stationary Vortex Instabilities in a Boundary-Layer Flow," In P.W. Duck and P. Hall (Eds.) Proceedings of IUTAM Symposium on Nonlinear Instability and Transition in ThreeDimensional Boundary Layers, Manchester, U.K., July 17-20, 1995, Kluwer Academic Publishers, 1996.

Cossu. C., and Brandt, L., "Stabilization of Tollmien-Schlichting Waves by Finite Amplitude Optimal Streaks in the Blasius Boundary Layer,” Phys. Fluids, Vol. 14, L57-L60, 2002.

Farrell, B.F., "Optimal Excitation of Perturbations in Viscous Shear Flows," Phys. Fluids, Vol. 31, p. 2093, 1988.

Fischer, P.F., Kruse, G.W., and Loth, F., "Spectral element methods for transitional flows in complex geometries," J. of Sci. Comput. Vol. 17, 1, pp. 81-98, 2002.

Gaster, M., Grosch, C.E., and Jackson, T.L., "The Velocity Field Created by a Shallow Bump in a Boundary Layer," Phys. Fluids, Vol. 6(9), pp. 3079-3085, 1994. 
Joslin, R.D. and Grosch. C.E., "Growth Characteristics Downstream of a Shallow Bump: Computations and Experiment," Phys. Fluids, Vol. 7(12), pp. 3042-3047, 1995.

Kendall, J.M., "Study of the Effects of Free-Stream Turbulence upon Disturbances in the Pre-Transitional Laminar Boundary Layer (Part 1I); Laminar Boundary Layer Distortion by Surface Roughness; Effect upon Stability," AFWAL-TR-82-3002, Part II, April 1982.

Kendall, J.M., "Experimental Study of Disturbances Produced in a Pre-Transitional Laminar Boundary Layer by Weak FreeStream Turbulence," AIAA Paper 85-1695, 1985.

Klebanoff, P., Cleveland, W.G., and Tidstrom, K.D., "On the Evolution of a Turbulent Boundary Layer Induced by a ThreeDimensional Roughness Element," J. Fluid Mech., Vol. 237, pp. 101-187, 1992. 1980.

Landahl, M.T., "A Note on an Algebraic Instability of Inviscid Parallel Shear Flows," J. Fluid Mech., Vol. 98, pp. 243-251,

Leib, S.J., Wundrow, D.W., and Goldstein, M.E., "Effect of Free-Stream Turbulence and Other Vortical Disturbances on a Laminar Boundary Layer," J. Fluid Mech., Vol. 380, pp. 169-203, 1999.

Luchini, P., "Reynolds-Number-Independent Instability of the Boundary Layer over a Flat Surface: Optimal Perturbations," J. Fluid Mech., Vol. 404, pp. 289-309, 2000.

Reshotko, E., "Transient Growth - A Factor in Bypass Transition," Phys. Fluids, Vol. 13(5), pp. 1067-1075, 2001.

Reshotko, E. and Tumin, A., "Investigation of the Role of Transient Growth in Roughness-Induced Transition," AIAA Paper 2002-2850, 2002.

Sedney, R., "A Survey of the Effects of Small Protuberances on Boundary-Layer Flows," AIAA J., Vol. 11, No. 6, pp. 782792,1973

Smith, F.T., Sykes, R.I., and Brighton, P.W.W., "Two-Dimensional Boundary Layer Encountering a Three-Dimensional Hump," J. Fluid Mech., Vol. 83, part 1. pp. 163-176, 1977.

Tani, I., Komoda, H., Komatsu, Y., and Iuchi, M., “Boundary-Layer Transition by Isolated Roughness," Rept. 375, Nov. 1962, Aero. Res. Inst., Univ. of Tokyo, Tokyo, Japan, pp. 129-142, Nov. 1962.

Trefethen L.N., Trefethen, A.E., Reddy, S.C., and Driscoll, T.A., "Hydrodynamic Stability Withouit Eigenvalues," Science, Vol. 261, pp. 578-84, 1993.

Tumin, A., and Reshotko, E., "Spatial Theory of Optimal Disturbances in Boundary Layers," Phys. Fluids, Vol. 13(7), pp. 2097-2104, 2001.

Tumin, A., and Reshotko, E., “The Problem of Boundary-Layer Flow Encountering a Three-Dimensional Hump Revisited," AIAA Paper 2004-0101, 2004.

Westin, K.J.A., Boiko, A.V., Klingmann, B.G.B., Kozlov, V.V., and Alfredsson, P.H., "Experiments in a Boundary Layer Subjected to Free-Stream Turbulence. Part 1. Boundary Layer Structure and Receptivity," J. Fluid Mech., Vol. 281, pp. 193-218, 1994.

White, E.B., "Transient Growth of Stationary Disturbances in a Flat Plate Boundary Layer," Phys. Fluids, Vol. 14(2), pp. $4429-4439,2002$.

White, E.B. and Reshotko, E., "Roughness-Induced Transient Growth in a Flat-Plate Boundary Layer," AIAA Paper 2002$0138,2002$.

White, E.B., and Ergin, F.G., "Receptivity and Transient Growth of Roughness-Induced Disturbances," AIAA Paper 2003$4243,2003$.

Woerner , A., Rist, U., and Wagner, S., "Investigation of the Flow in the Vicinity of an Isolated 3D Surface Roughness," In: C. Breitsamter, B. Laschka, H.J. Heinemann, R. Hilbig (Eds.): New Results in Numerical and Experimental Fluid Mechanics IV, Contributions to the 13th STAB/DGLR Symposium, Munich, Germany 2002, NNFM 87, Springer-Verlag, pp. $319-326,2004$.

Wu, X., and Luo, J., "Linear and Nonlinear Instabilities of a Blasius Boundary Layer Perturbed by Streamwise Vortices. Part 1. Steady Streaks," J. Fluid Mech., Vol. 483, pp. 225-248, 2003. 


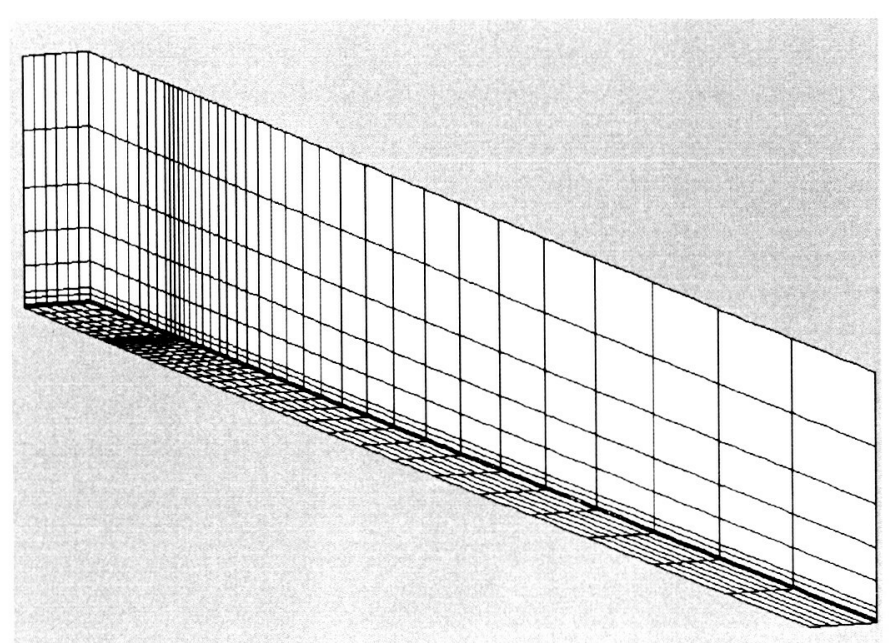

(a) Schematic of the combined near- and far-zone grids.

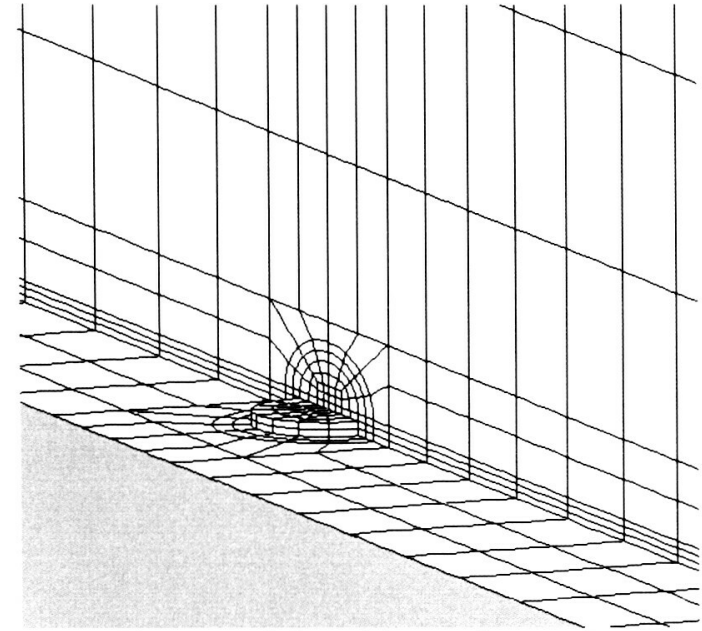

(b) Close-up of the grid near the roughness element, including a symmetry-plane cut.

Figure 1. Spectral element mesh.
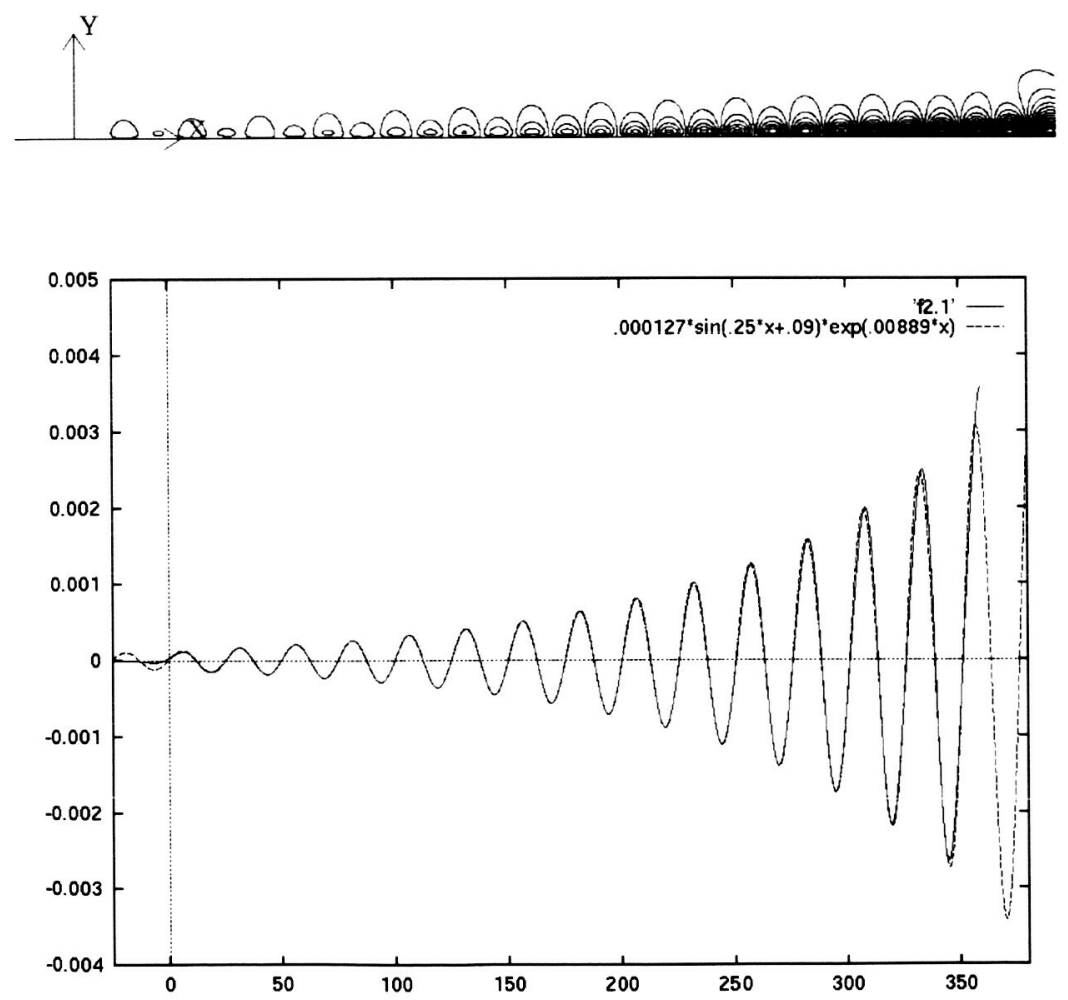

Figure 2. Validation of spectral element code for Tollmien-Schlichting wave propagation $\left(\mathrm{F}=70 \times 10^{-6}\right)$. Top: contours of v' downstream of source (an unsteady suction-blowing strip at $\operatorname{Re}_{x}=0.5 \times 10^{6}$ ). Bottom: Magnitude of v' vs. $x$ at $y=1$, compared with linear stability theory (dashed line). 


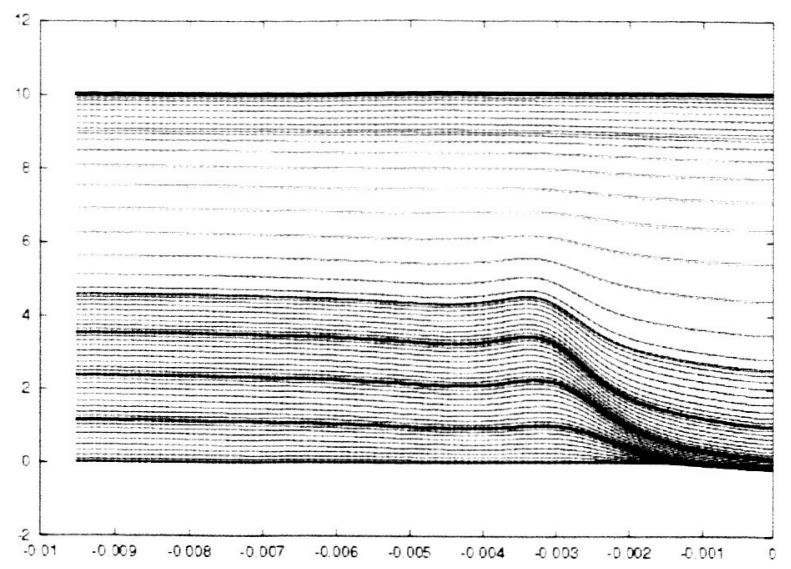

(a) u profiles

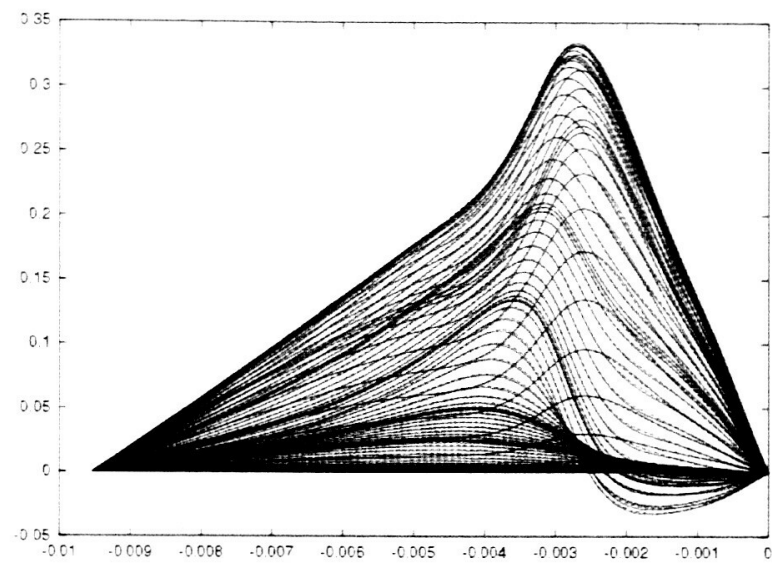

(b) w profiles

Figure 3. Grid convergence of nearfield solution for largest height roughness array $(\mathrm{h}=0.665 \mathrm{~mm}$ ): velocity profiles at $\mathrm{x}-\mathrm{x}_{0}=0.008 \mathrm{~m}$. Velocity components $\mathrm{u}$ and $\mathrm{w}$ (measured in $\mathrm{m} / \mathrm{s}$ ) are plotted as functions of spanwise coordinate $\mathrm{z}$ (in meters) for various heights above the surface. Solid lines denote $\mathrm{N}=11$ solution, whereas dashed lines indicate the $\mathrm{N}=7$ solution.

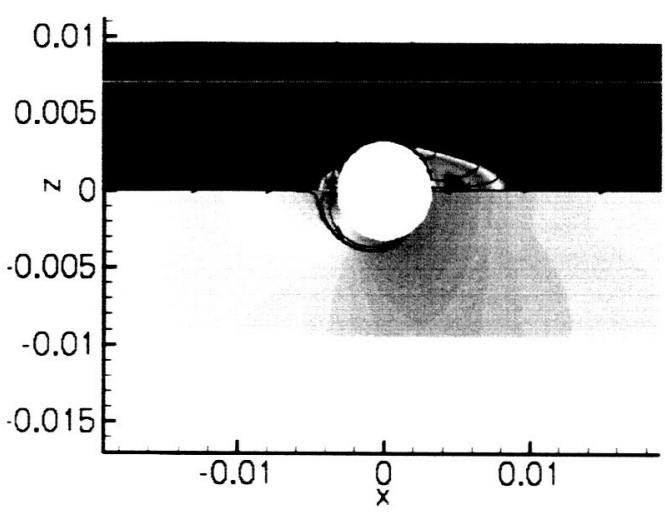

Figure 4. Flow features near roughness array: surface pressure distribution (lower half) and streamwise shear stress (upper half). Warmer shades denote peak pressures or large positive shear stress; cooler shades denote the opposite conditions.

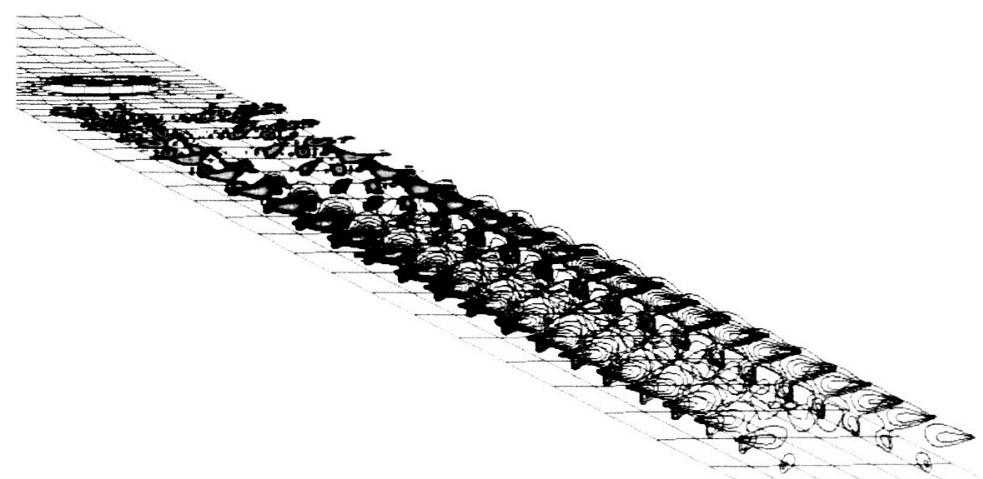

Figure 5. Stationary vortex structures in the wake of spanwise periodic roughness array (baseline configuration).

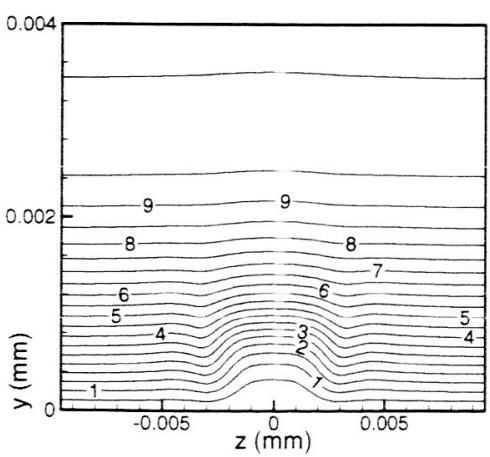

(a) $\mathrm{x}-\mathrm{x}_{0}=10 \mathrm{~mm}$

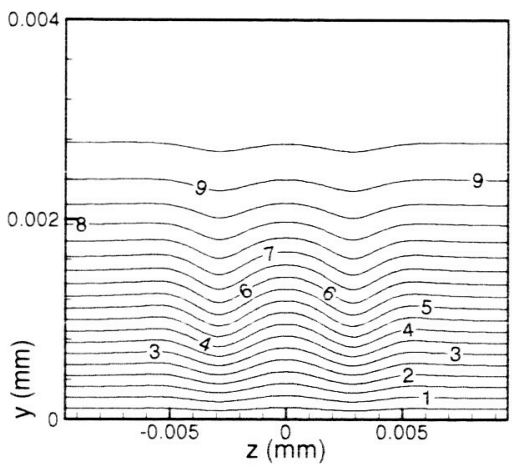

(a) $\mathrm{x}-\mathrm{x}_{0}=80 \mathrm{~mm}$

Figure 6. Streamwise velocity contours at $x-x_{0}=10 \mathrm{~mm}$ and $80 \mathrm{~mm}$, respectively (contour labels indicate velocity in $\mathrm{m} / \mathrm{s}$ ). 


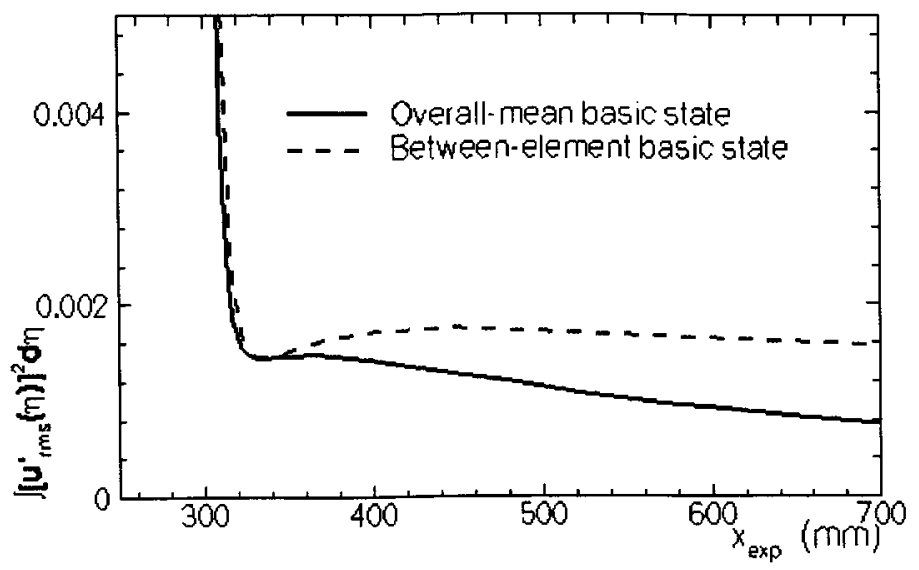

Figure 7. Evolution of total disturbance energy behind baseline roughness array $(\mathrm{h}=0.57 \mathrm{~mm})$.

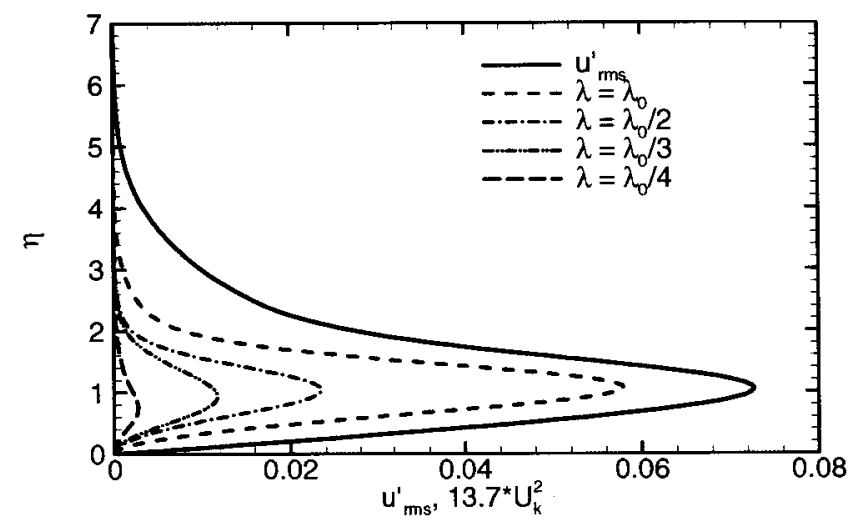

(a) $\mathrm{x}-\mathrm{x}_{0}=10 \mathrm{~mm}$

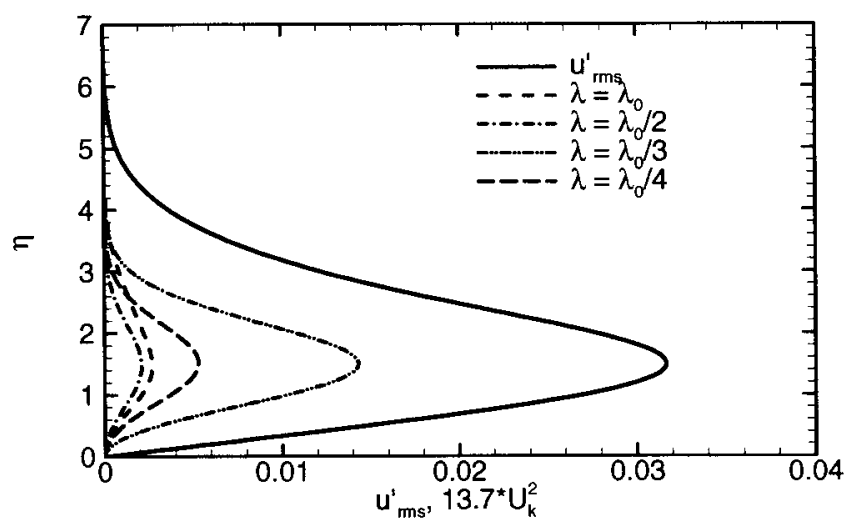

(a) $\mathrm{x}-\mathrm{x}_{0}=80 \mathrm{~mm}$

Figure 8. RMS and modal disturbance profiles at $\mathrm{x}-\mathrm{x} 0=10 \mathrm{~mm}$ and $80 \mathrm{~mm}$, respectively. The modal profiles correspond to square of the peak amplitude in the corresponding Fourier component, scaled by a constant factor of 13.7 .

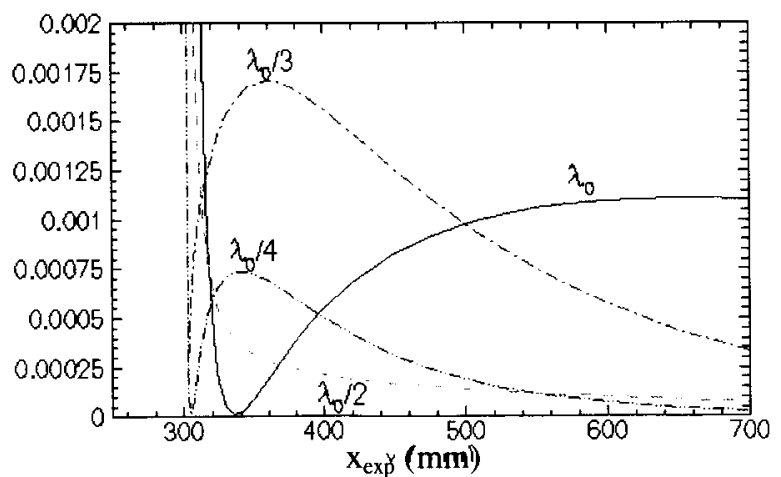

(a) Modal energies $\left.\int \mathrm{U}_{\mathbf{k}}{ }^{2}(\eta) \mathrm{d \eta}\right)$ based on simulation

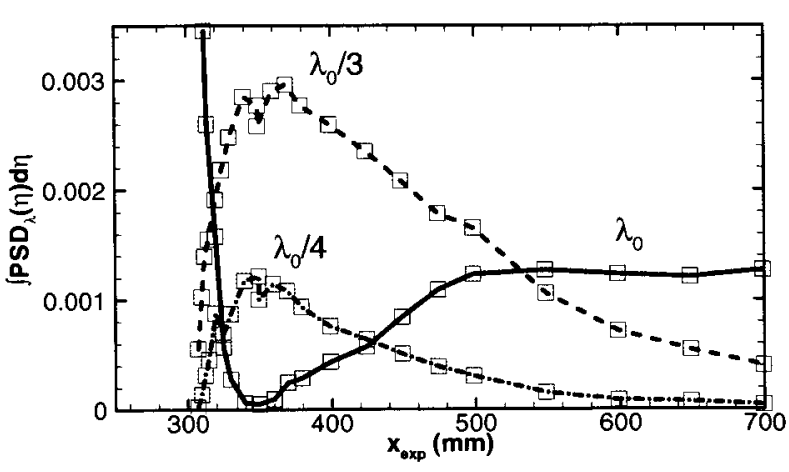

(a) Measured data from White and Ergin (2003)

Figure 9. Streamwise evolution of modal energy in dominant Fourier components. 


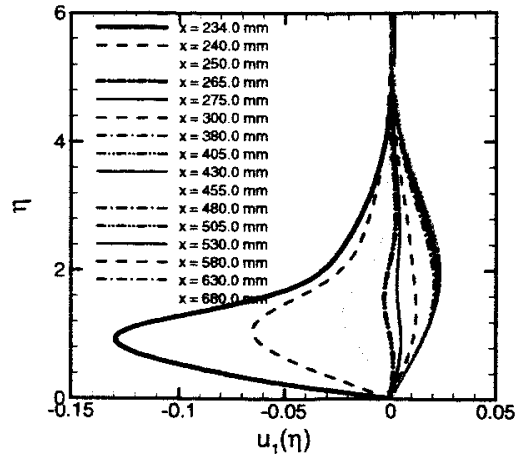

(a) $U_{1}(\eta)$

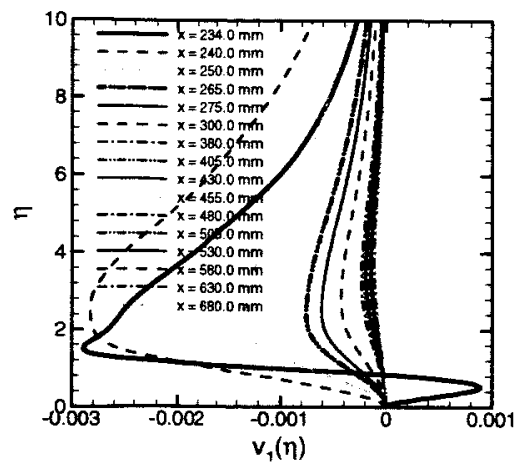

(b) $V_{1}(\eta)$

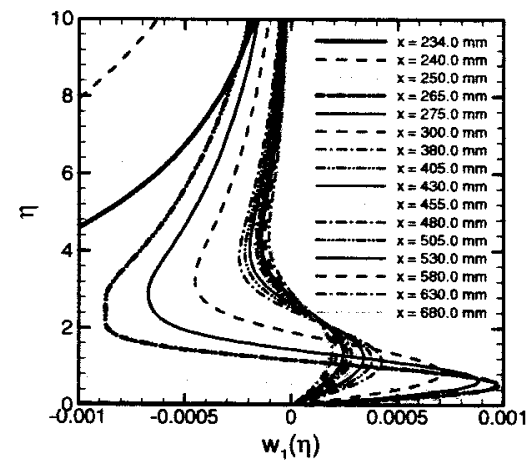

(c) $\mathrm{W}_{1}(\eta)$

Figure 10. Modal profiles for the fundamental spanwise mode at selected streamwise locations behind roughness array $(\mathrm{h}=0.57 \mathrm{~mm})$.

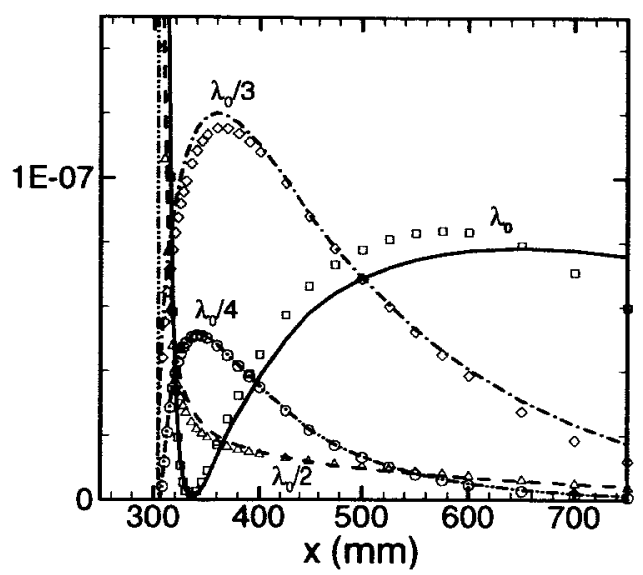

Figure 11. Effect of roughness height on transient growth: normalized modal energies $E_{k}(x) / \operatorname{Re}_{k}{ }^{2}(k=1-4)$, plotted as functions of $x$ for baseline array $\left(\operatorname{Re}_{\mathrm{k}}=119\right)$ and the larger-height array with $\operatorname{Re}_{\mathrm{k}}=162$. 